\title{
Behavioral and physiological methods for early quantitative assessment of spinal cord injury and prognosis in rats
}

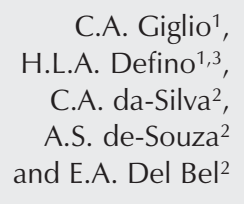

C.A. Giglio ${ }^{1}$,

H.L.A. Defino ${ }^{1,3}$,

C.A. da-Silva²,

A.S. de-Souza ${ }^{2}$ and E.A. Del Bel ${ }^{2}$

\author{
${ }^{1}$ Escola de Engenharia de São Carlos, Universidade de São Paulo, \\ São Carlos, SP, Brasil \\ ${ }^{2}$ Departamento de Morfologia, Estomatologia e Fisiologia, \\ Faculdade de Odontologia de Ribeirão Preto, \\ ${ }^{3}$ Departamento de Biomecânica, Medicina e Reabilitação do Aparelho \\ Locomotor, Faculdade de Medicina de Ribeirão Preto, \\ Universidade de São Paulo, Ribeirão Preto, SP, Brasil
}

\section{Correspondence \\ E.A. Del Bel \\ Departamento de Morfologia, \\ Estomatologia e Fisiologia \\ FORP, USP \\ Av. do Café, s/n \\ 14049-904 Ribeirão Preto, SP \\ Brasil \\ Fax: +55-16-3633-0999 \\ E-mail: eadelbel@forp.usp.br}

Part of a Master's thesis presented

by C.A. Giglio to the Faculdade de

Medicina de Ribeirao Preto, and

Escola de Engenharia de São Carlos, IQSC, USP.

Publication supported by FAPESP, CNPq and CAPES.

Received October 30, 2005 Accepted September 26, 2006

\begin{abstract}
Methods for reliable evaluation of spinal cord (SC) injury in rats at short periods ( 2 and $24 \mathrm{~h}$ ) after lesion were tested to characterize the mechanisms implicated in primary SC damage. We measured the physiological changes occurring after several procedures for producing SC injury, with particular emphasis on sensorimotor functions. Segmental and suprasegmental reflexes were tested in 39 male Wistar rats weighing 250-300 g divided into three control groups that were subjected to a) anesthesia, b) dissection of soft prevertebral tissue, and c) laminectomy of the vertebral segments between $T_{10}$ and $L_{1}$. In the lesion group the SC was completely transected, hemisected or subjected to vertebral compression. All animals were evaluated 2 and 24 $\mathrm{h}$ after the experimental procedure by the hind limb motility index, Bohlman motor score, open-field, hot-plate, tail flick, and paw compression tests. The locomotion scale proved to be less sensitive than the sensorimotor tests. A reduction in exploratory movements was detected in the animals $24 \mathrm{~h}$ after the procedures. The hot-plate was the most sensitive test for detecting sensorimotor deficiencies following light, moderate or severe SC injury. The most sensitive and simplest test of reflex function was the hot-plate. The hemisection model promoted reproducible moderate SC injury which allowed us to quantify the resulting behavior and analyze the evolution of the lesion and its consequences during the first $24 \mathrm{~h}$ after injury. We conclude that hemisection permitted the quantitation of behavioral responses for evaluation of the development of deficits after lesions. Hind limb evaluation scores and spontaneous exploration events provided a sensitive index of immediate injury effects after SC lesion at 2 and 24 h. Taken together, locomotion scales, open-field, and hot-plate tests represent reproducible, quantitatively sensitive methods for detecting functional deficiencies within short periods of time, indicating their potential for the study of cellular mechanisms of primary injury and repair after traumatic SC injury.
\end{abstract}

Key words

- Trauma

- Spinal cord injury

- Experimental models

- Sensorial test

- Motor performance test

..................... 


\section{Introduction}

Spinal cord (SC) injury causes serious consequences that can persist for an entire life span $(1,2)$. One objective of SC injury research is to understand the post-lesion events so as to minimize injury or perhaps devise strategies for repairing damage. It is known that the immature mammalian central nervous system can repair itself after injury, but only up to a particular stage of development after which the capacity for repair is lost $(2,3)$. In contrast, in adults peripheral nerves regenerate successfully with recovery of function after injury $(4,5)$.

Mechanical force applied to the SC causes primary damage to neural tissue. Over a period of minutes to hours the affected area spreads through complex cascades that give rise to secondary damage $(2,4,5)$. The acute post-injury phase is critically important for recovery $(4,6)$. The injured nerve cells respond with an injury-induced barrage of action potentials. Accompanying this are significant electrolytic shifts, principally involving monovalent $\left(\mathrm{Na}^{+}, \mathrm{K}^{+}\right)$and divalent cations $\left(\mathrm{Ca}^{2+}\right)(1,2,4)$. Increases in intracellular $\mathrm{Ca}^{2+}$ to toxic levels contribute to failure of normal neural functions. Spinal shock, which lasts for about $24 \mathrm{~h}$ in rats, represents a generalized failure of circuitry in the spinal neural network $(2,4,7)$. Apart from the direct effects on neural tissues, hemorrhage, localized edema, thrombosis, vasospasm, and loss of vasculature autoregulation all contribute to the damage, in addition to the compression of the SC that follows vertebral displacement and edema (79). In the best circumstances the time to admission after SC injury is usually not less than $3 \mathrm{~h}$ $(1,2)$. Hence there is little information about the immediate effects of acute injury. The extent of our understanding is reflected in the limited neuroprotective strategies currently available beyond rapid trauma resuscitation and attentive clinical care $(1,2,4)$.

At present there is no simple or well-defined procedure available for complete char- acterization of the severity of SC injury. One experimental procedure is contusion of the $\mathrm{SC}$ $(6,7)$, which mimics the situation in humans. However, with this approach it is not possible to interrupt specific and well-defined spinal tracts in the same way as done by sectioning at specific locations (5). Other less clinically relevant models consist of compression $(8,9)$, transection (10) or hemisection (11). Transection has the distinct advantage of consistent reproducibility. Hemisection permits to compare cellular responses ipsi- and contra-lateral to the injury. An extensive review of experimental studies postulates that permanent paraplegia induced in rats represents an appropriate experimental model. Therefore, each lesion mimics a particular aspect of the damage to the SC that occur in humans $(12,13)$.

The development of new animal models should provide strategies to help patients with SC injuries to be evaluated in terms of residual motor and sensory function. Accordingly, we have quantitatively investigated differences in behavior following SC injury within 2 and $24 \mathrm{~h}$ after lesioning in order to characterize the mechanisms implicated in primary SC damage.

\section{Material and Methods}

\section{Animals}

Thirty-nine male Wistar rats weighing 250-300 $\mathrm{g}$ were kept in a temperature-controlled room $\left(23^{\circ} \mathrm{C}\right)$ with a 12 -h light/dark cycle (lights on at 7:00 am) with free access to water and food. The experiments were carried out according to the guidelines of the Brazilian Society of Neuroscience and Behavior for the care and use of laboratory animals. All efforts were made to minimize animal suffering. Experiments were performed between 8:00 and 12:00 am.

\section{Surgical preparation}

All surgical techniques were performed 
under aseptic conditions. Animals were anesthetized by intraperitoneal injection of 250 $\mathrm{mg} / \mathrm{kg}$ 2,2,2-tribromoethanol (Aldrich Chemical Company Inc., Milwaukee, WI, USA). After shaving the skin, animals were kept in a David Kopf stereotaxic apparatus and an incision was made in the skin and underlying muscles of the mid-thoracic region (14). Muscles were retracted and a mid-thoracic laminectomy was performed (vertebral segments $\mathrm{T}_{10}-\mathrm{L}_{1}$ ). When required, the dura mater was exposed. The animals were divided into three control groups and three test groups and then submitted to different types of procedures.

\section{Spinal cord injury}

Control groups. Anesthesia. Control unoperated rats $(\mathrm{N}=4)$ were anesthetized for the same periods of time as needed for the surgical procedures.

Removal of soft tissue. A dorsal incision was made in the skin and underlying muscles of anesthetized rats $(\mathrm{N}=9)$. Muscles were retracted $\left(\mathrm{T}_{10}-\mathrm{L}_{1}\right)$ and muscular tissue was removed to expose the vertebral bone plate which was left intact.

Laminectomy. The vertebra was approached as described above and vertebral bone of the $\mathrm{T}_{10}-\mathrm{L}_{1}$ segments (laminectomy) was removed bilaterally to expose the dura mater, which was left intact $(\mathrm{N}=12)$.

Test groups. Complete SC section. After laminectomy, the dura mater was opened and a sharp tiny blade $(0.2 \mathrm{~mm})$ was positioned perpendicularly to the tissue, touching the inferior side of the bone plate. A complete cut of the cord was made with the blade running completely from left to right, twice $(\mathrm{N}=6)$.

Hemisection of the right side of the $S C$. Anesthetized animals $(\mathrm{N}=4)$ were submitted to a procedure similar to that described above. To produce this lesion, the blade $(0.2$ $\mathrm{mm}$ ) was positioned perpendicularly to the tissue, touching the inferior side of the bone plate. A cut of the cord was made with the blade running carefully from the right side to the midline of the cord.

Vertebral compression. Based on the method of Gruner et al. (9), the tips of a hemostat forceps were inserted between the spine and the surrounding muscle of each rat $(\mathrm{N}=4)$. The spine was compressed between the $\mathrm{T}_{10}-\mathrm{L}_{1}$ segments by slowly closing the arms of the forceps and holding them closed for $15 \mathrm{~s}$.

The injury sites were irrigated with saline and the incisions were closed in layers using silk sutures (4-0 thread, Ethicon, São Jose dos Campos, SP, Brazil). After receiving penicillin subcutaneously, the animals were placed in a warming chamber in individual boxes and body temperature was maintained at $37^{\circ} \mathrm{C}$ until they were fully awake.

After the end of the behavioral tests (2 and $24 \mathrm{~h}$ after surgery), rats were sacrificed with a lethal dose of anesthetic. The lesion site $\left(\mathrm{T}_{10}-\mathrm{L}_{1}\right)$ was confirmed by macroscopic and microscopic observation.

\section{Behavioral tests of functional deficits}

Behavioral data were collected from the rats after dorsal hemisection, complete SC section and vertebral compression or control procedures (anesthesia, removal of tissue and laminectomy groups). We used motor and sensory tasks and quantitative evaluation according to defined scaling systems. The animals were evaluated 2 and $24 \mathrm{~h}$ after each procedure. The battery of experimental behavioral tests was applied once to each animal in the same order and consisted of: 1) hind limb motility index, 2) Bohlman motor evaluation score, 3) open-field, 4) hot-plate, 5) tail flick, and 6) paw compression tests. A varying number of animals per test were analyzed since data were collected from control animals (laminectomy) in several experiments.

Hind limb motility index. The qualitative aspects of the reaching movements of each animal were analyzed by assessing the abil- 
ity of the hind limbs to move over a period of 2 min (8). Animals were placed on a smooth flat surface and allowed to walk freely. The following scores were applied: $0=$ normal moving ability of the hind limbs, $1=$ paresis or decreased moving ability of the hind limbs, $2=$ plegia or blocked moving ability of the hind limbs.

Bohlman motor evaluation score. A commonly used instrument is the Tarlov scale (15) which ranks hind limb movements and weight support. The Tarlov score was modified by Bohlman (16). Animals were evaluated and assigned to one of the five categories: grade 0 , complete paraplegia without movements; grade 1, small articulate movements; grade 2, large movements; grade 3, standing on their feet; grade 4, walking; grade 5, walking on a plane with a 20-degree inclination. Scores 0-4 were attributed after observing the rats on a smooth flat surface for $4 \mathrm{~min}$. Orientation of the body in space was measured by using a $20^{\circ}$ inclined board ( $1 \mathrm{~m}$ long, $0.5 \mathrm{~m}$ wide and $0.02 \mathrm{~m}$ width) covered with rubber. Both the time to orient the body to face upward and the motor patterns used to turn the body around were recorded. The rat was placed with all paws on the board, with its body and nose facing down (10). No obvious signs of pain or stress were observed in any group.

Open-field test. To assess exploratory behavior $(17,18)$, rats were tested in an openfield consisting of a circular wooden box (72 $\mathrm{cm}$ in diameter) surrounded by 49-cm high transparent walls with an open top. The floor was divided into twelve equal size fields. Behavioral activity was measured for periods of 4 min at the beginning of the dark cycle (10:00 am to $11: 30 \mathrm{pm}$ ) at 2 and $24 \mathrm{~h}$ after surgery. Each activity chamber was cleaned with alcohol between tests to eliminate urine and olfactory cues from previous subjects.

Each rat was gently placed in the center of the open-field and hand-operated counters and stopwatches were used to score behaviors. Four different behavioral measures of sponta- neous activity were determined: locomotion or exploratory movement, measured as the number of floor units entered with the four paws; rearing frequency, as the number of times the animal stood on its hind limbs; grooming or stationary movement frequency, and defecation. The four parameters of spontaneous behaviors were empirically found to be best for measurements of individual and group behaviors. The number of rearing events is important for analyzing the effect of laminectomy alone (sham) versus spinal injury. Exploratory measurements are necessary since stationary activity may not reflect the animal's exploratory behavior in the field in an accurate manner. We evaluated changes in each parameter individually. A white noise generator provided a constant background noise.

Hot-plate test. The animals were placed on a hot-plate maintained at $52 \pm 0.5^{\circ} \mathrm{C}$. The thermal nociceptive threshold was defined as the time required to elicit a hind paw lick or a jump (14). The cut-off time was $20 \mathrm{~s}$.

Tail flick test. Spinal reflexes were assessed by the tail flick test. Each rat was placed in a ventilated glass tube with the tail laid across a nickel-chrome wire coil maintained at room temperature $\left(23 \pm 2^{\circ} \mathrm{C}\right)$. The coil temperature was then raised at the rate of $9^{\circ} \mathrm{C} / \mathrm{s}$ by the passage of an electric current, adjusted to ensure a tail withdrawal reflex within 2.5 to $3.5 \mathrm{~s}$. A cut-off time of $6 \mathrm{~s}$ was established to minimize skin damage (14). Animals were tested for 3 consecutive trials. Antinociception measurements were made before and after the experimental procedures.

Paw compression test. Hind paw hyperalgesia was measured as described by Randall and Selitto (19), using an Ugo Basile analgesia-meter (Stoelting Co., Wood Dale, IL, USA). A plastic device was in contact with the $3 \mathrm{rd}$ interdigital space of the hind legs, which were lying on a plane surface. The progressive weight was then transferred to the 3rd interdigital rod. The test was stopped when the animal performed any movement of the paw or when the weight reached a maximal level. 
The force at which a rat withdrew its hind paw or struggled was multiplied by 10 , as recommended by the manufacturer, and recorded as the withdrawal force $(\mathrm{g})$.

\section{Statistical analysis}

Well-established scoring methods used for human neurological examinations were used to evaluate and classify symptoms. The non-parametric Kruskal-Wallis test was used for the hind leg motility index and Bohlman motor evaluation score and multivariate analysis of variance (MANOVA) was used for the open-field, hot-plate, tail flick, and paw compression tests. The items analyzed were procedure, time and procedure $x$ time interaction. In cases of significance of the procedure-time interaction, ANOVA was employed, followed by the Duncan post hoc test. The level of significance was set at $\mathrm{P}<$ 0.05 for all tests.

\section{Results}

\section{Hind limb motility index}

The median scores attributed to the animals of each group are shown in Table 1.
The animals belonging to the anesthesia, laminectomy, removed soft tissue, complete section, hemisection, and vertebral compression groups received a single score for each group, suggesting homogeneity of the experimental procedure and effects. A score of two for the complete section and hemisection groups denotes paralysis $\left(2 \mathrm{~h}: \chi^{2}=\right.$ 66.00, d.f. $=6, \mathrm{P}<0.001 ; 24$ h: $\chi^{2}=61.99$, d.f. $=6, \mathrm{P}<0.001)$.

\section{Bohlman score}

Table 1 also shows the results obtained for the inclined plane and their Bohlman scores. All animals in the anesthesia, removed soft tissue and vertebral compression groups could walk 2 or $24 \mathrm{~h}$ after treatment. Nine of 12 animals from the laminectomy group were able to walk, but their ability was lower for the 24-h period than for the 2-h period. Animals from the complete section and hemisection groups lost the ability to move down on the inclined plane. Therefore, the results of the inclined plane test differed significantly between the groups anesthesia, removed soft tissue, laminectomy, and compression and the groups complete section and hemisection $\left(2 \mathrm{~h}: \chi^{2}=\right.$

Table 1. Motility index for fore- and hind legs of rats after spinal cord lesion.

\begin{tabular}{|c|c|c|c|c|c|c|}
\hline Test & $\begin{array}{l}\text { Anesthesia } \\
\quad(N=4)\end{array}$ & $\begin{array}{l}\text { Removed soft } \\
\text { tissue }(\mathrm{N}=9)\end{array}$ & $\begin{array}{l}\text { Laminectomy } \\
\qquad(\mathrm{N}=12)\end{array}$ & $\begin{array}{c}\text { Complete } \\
\text { section }(N=6)\end{array}$ & $\begin{array}{l}\text { Hemisection } \\
\quad(\mathrm{N}=4)\end{array}$ & $\begin{array}{l}\text { Compression } \\
(\mathrm{N}=4)\end{array}$ \\
\hline \multicolumn{7}{|c|}{ Bohlman score } \\
\hline $2 \mathrm{~h}$ & 5 & 5 & 5 & 0 & 0 & 5 \\
\hline $24 \mathrm{~h}$ & 5 & 5 & 4 & 0 & 0 & 5 \\
\hline \multicolumn{7}{|c|}{ Inclined plane } \\
\hline $2 \mathrm{~h}$ & + & + & + & - & - & + \\
\hline $24 \mathrm{~h}$ & + & + & - & - & - & + \\
\hline \multicolumn{7}{|c|}{ Hind limb motility index } \\
\hline $2 \mathrm{~h}$ & 0 & 0 & 1 & 2 & 2 & 0 \\
\hline $24 \mathrm{~h}$ & 0 & 0 & 1 & 2 & 2 & 0 \\
\hline
\end{tabular}

Movements of fore- and hind limbs were analyzed during 5-min periods 2 and $24 \mathrm{~h}$ after treatment and were measured using the hind limb motility index: 0: normal movement of the hind legs; 1: paresis - decreased movement of the hind legs; 2: plegia - blocked movement of the hind legs. Bohlman score: $0=$ complete paraplegia, without movements; 1 = small articulated movements; 2 = large movements; 3 = animal can stand on its feet; $4=$ animal can walk; $5=$ animal can walk on a $20^{\circ}$ inclined plane. 
33.00 , d.f. $=6, \mathrm{P}=0.005 ; 24$ h: $\chi^{2}=25.32$, d.f. $=6, \mathrm{P}<0.001)$.

The animals in the anesthesia, removed soft tissue, and vertebral compression groups scored 5, a normal Bohlman score, whereas the animals in the laminectomy group showed partial motor losses. Those in the complete section and hemisection groups had total loss of motor functions, with the lowest Bohlman score $\left(2 \mathrm{~h}: \chi^{2}=21.46\right.$, d.f. $=6, \mathrm{P}=$

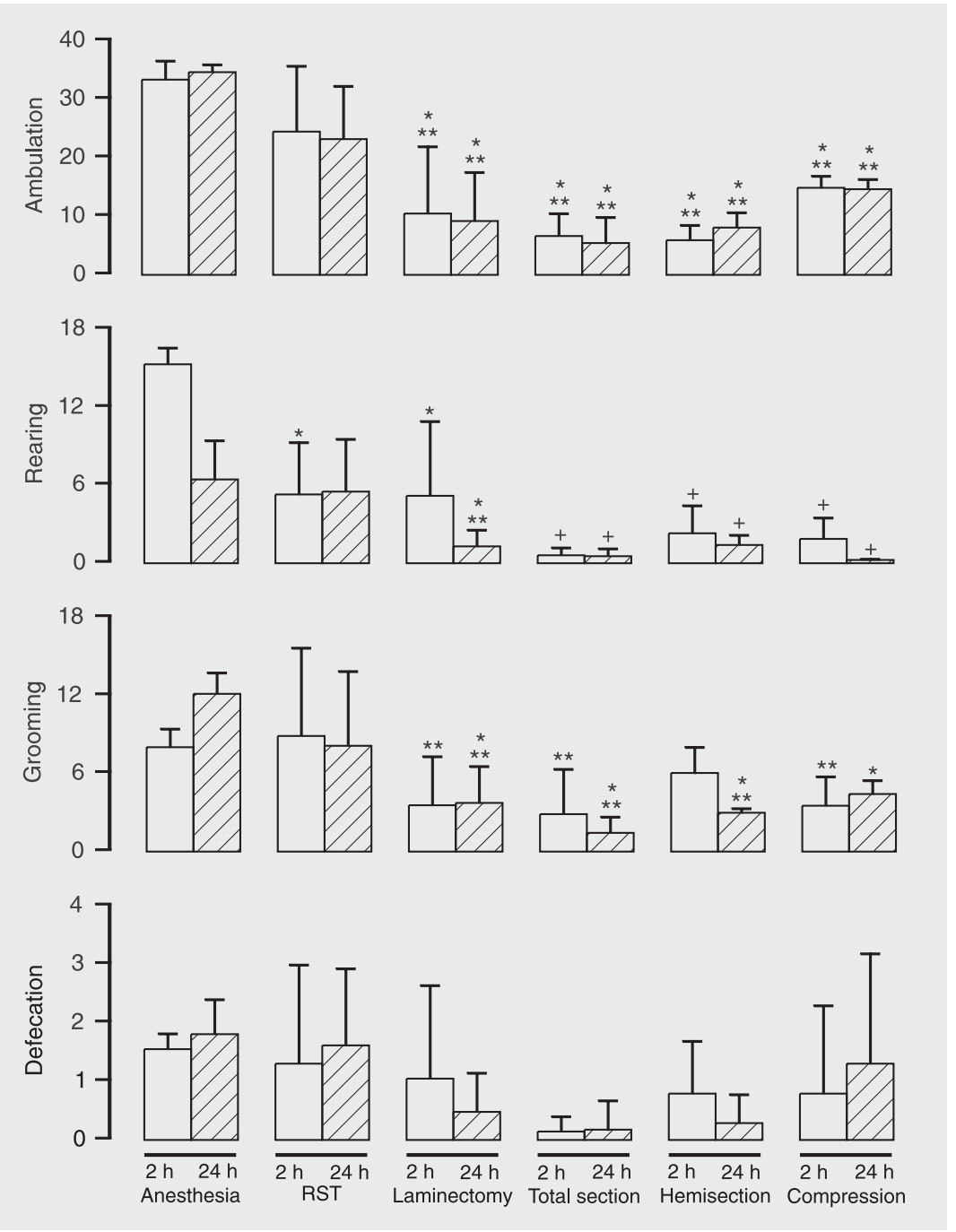

Figure 1. Effects of spinal cord lesion on exploratory behavior of rats tested in an open-field. Ambulation, rearing, grooming, and defecation were recorded for $5 \mathrm{~min}$ and analyzed $2 \mathrm{~h}$ (open bars) and $24 \mathrm{~h}$ (hatched bars) after treatment. Data are reported as the means \pm SEM. ${ }^{*} \mathrm{P}<0.05$ compared to anesthesia (control); ${ }^{* *} \mathrm{P}<0.05$ compared to removed soft tissue (RST); ${ }^{+} \mathrm{P}<0.05$ compared to the anesthesia (control), RST and laminectomy groups (ANOVA followed by the Duncan test for all comparisons). For animal treatment specification and number of rats in each group, see Table 1.
$0.005 ; 24$ h: $\chi^{2}=22.28$, d.f. $=6, P=0.001$ ). No signs of pain were seen in any group.

\section{Open-field test}

Quantitative march analysis (ambulation) for the evaluation of locomotion ability is shown in Figure 1.

The laminectomy, complete section, hemisection, and vertebral compression groups caused a significantly reduced numbers of floor units to be crossed when compared with the anesthesia and removed soft tissue groups at both 2 and $24 \mathrm{~h}$ after surgery (ANOVA; 2 h: $\mathrm{F}_{6,26}=6.68, \mathrm{P}<0.001,24 \mathrm{~h}$ : $\left.\mathrm{F}_{6,26}=13.21, \mathrm{P}<0.001\right)$. In assays performed $2 \mathrm{~h}$ after surgery, there was a similar decrease in the number of quadrants crossed by the animals in the laminectomy, complete section, hemisection, and vertebral compression groups compared with the anesthesia and removed soft tissue groups. For the 24-h period, the anesthesia and removed soft tissue groups crossed a larger number of quadrants compared with other groups. The anesthesia group also crossed a larger number of quadrants than the removed soft tissue group.

The number of rearing events was decreased in most experimental groups compared with the anesthesia group. There was a significant general effect of lesion $\left(\mathrm{F}_{6,26}=\right.$ 9.32, $\mathrm{P}=0.001$ ) and an interaction between lesion and time $\left(\mathrm{F}_{6,26}=10.93, \mathrm{P}<0.001\right)$. This decrease was statistically significant by ANOVA (lesion, $2 \mathrm{~h}: \mathrm{F}_{6,26}=9.32, \mathrm{P}<0.001$; $\left.24 \mathrm{~h}: \mathrm{F}_{6,26}=5.66, \mathrm{P}=0.001\right)$ for the removed soft tissue ( 2 h only), laminectomy, complete section, hemisection, and vertebral compression groups compared with the anesthesia group.

A decrease in the number of grooming events was observed in most experimental groups compared with the anesthesia and removed soft tissue groups. There was a significant effect of lesion $\left(\mathrm{F}_{6,26}=5.47, \mathrm{P}=\right.$ $0.001)$ but not of time $\left(\mathrm{F}_{6,26}=1.07, \mathrm{P}=0.31\right)$. No interaction between lesion and time $\left(\mathrm{F}_{6,26}\right.$ 
$=1.74, \mathrm{P}=0.15)$ was found. This decrease was statistically significant by ANOVA (lesion, 2 h: $\mathrm{F}_{6,26}=3.24, \mathrm{P}=0.005 ; 24 \mathrm{~h}: \mathrm{F}_{6,26}=$ $5.83, \mathrm{P}<0.001$ ) for the laminectomy, complete section, hemisection, and vertebral compression groups compared with the anesthesia and removed soft tissue groups.

No significant effect on defecation was detected in any experimental group (lesion: $\mathrm{F}_{6,26}=1.30, \mathrm{P}=0.29$; time: $\mathrm{F}_{6,26}=0.06, \mathrm{P}=$ 0.80 ; lesion $\mathrm{x}$ time: $\mathrm{F}_{6,26}=1.96, \mathrm{P}=0.11$ ).

\section{Tail flick test}

The results of this assay, used to measure thermal sensitivity by medullar reflexes, are shown in Figure 2. One-way ANOVA of the mean values revealed a significant effect of lesion $\left(\mathrm{F}_{6,26}=9.8, \mathrm{P}<0.001\right)$ but not of time $\left(\mathrm{F}_{6,26}=3.48, \mathrm{P}=0.073\right)$. No interaction between the lesion and time $\left(\mathrm{F}_{6,26}=0.23, \mathrm{P}=\right.$ 0.96) was found. Post hoc analysis showed that the animals subjected to anesthesia, removed soft tissue, laminectomy, and vertebral compression had significantly increased tail flick reactions compared to the complete section group at $2 \mathrm{~h}\left(\mathrm{~F}_{6,26}=7.32, \mathrm{P}<0.001\right)$. At $24 \mathrm{~h}$, the total section group had a longer lag time $\left(\mathrm{F}_{6,26}=6.32 ; \mathrm{P}<0.001\right)$, as did the hemisection group. Thus, the tail flick tests revealed a decreased ability of the total section and hemisection groups to respond to the thermal stimuli.

\section{Hot-plate test}

The results of the hot-plate test used to evaluate medullar and brain reflexes are also shown in Figure 2. Statistical analysis indicated significant effects of the different procedures (lesion: $\mathrm{F}_{6,26}=7.38, \mathrm{P}<0.001$ ). There was no time dependence $\left(\mathrm{F}_{6,26}=1.88\right.$, $P=0.31)$ and only marginal significance of the interaction between the type of injury and elapsed time $\left(\mathrm{F}_{6,26}=2.20, \mathrm{P}=0.075\right)$. After $2 \mathrm{~h}$, only the total section and vertebral compression groups differed from the anes- thesia and removed soft tissue groups $\left(\mathrm{F}_{6,26}=\right.$ $2.68, \mathrm{P}<0.005)$. However, $24 \mathrm{~h}$ after surgery, all groups differed from the anesthesia and removed soft tissue groups $\left(\mathrm{F}_{6,26}=23.76\right.$, $\mathrm{P}<0.001)$. Accordingly, the hot-plate tests showed function defects in all groups when compared with controls.

\section{Paw compression test}

Figure 3 shows the pain sensitivity re-

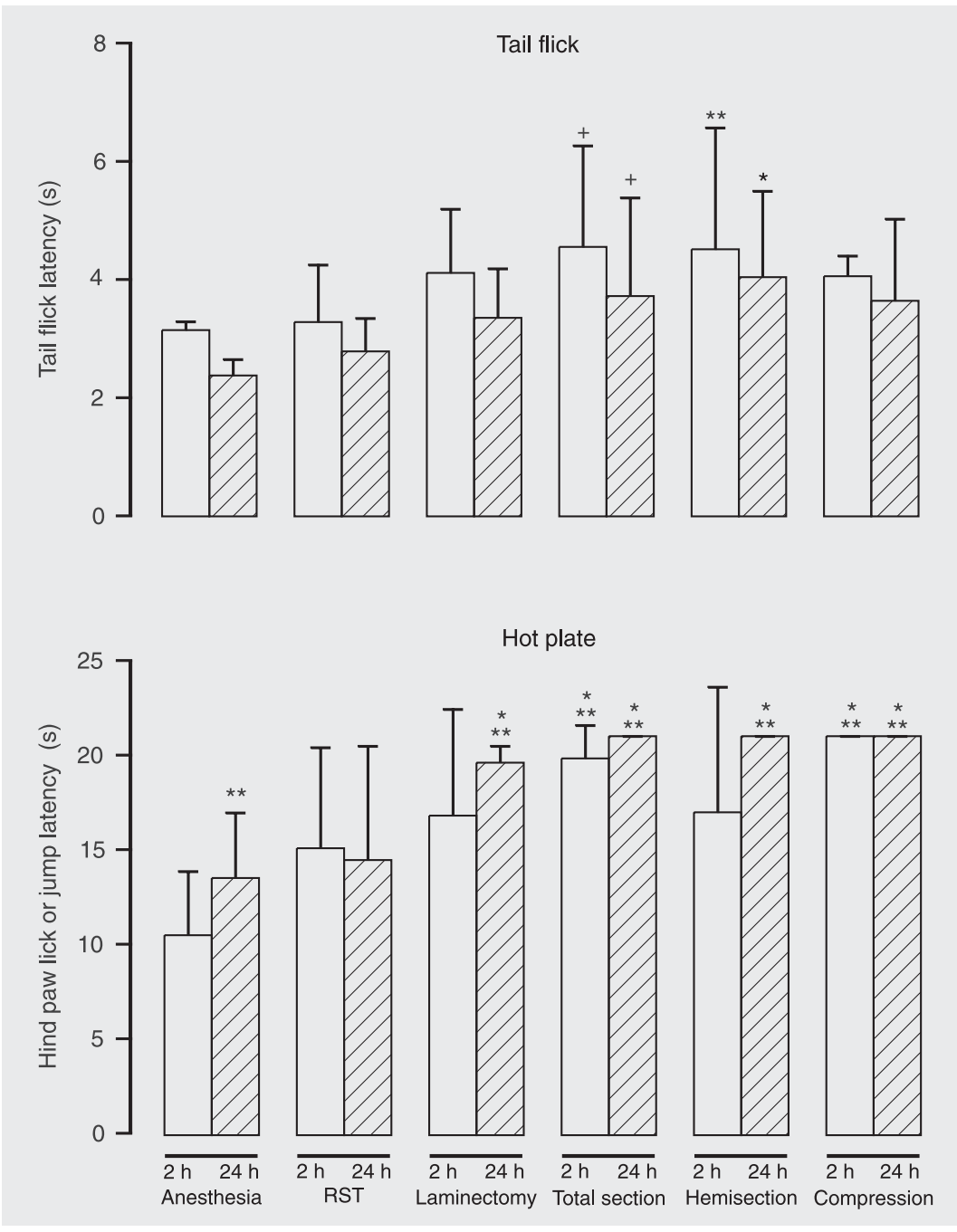

Figure 2. Effects of spinal cord lesion on sensitivity to pain tested by the tail flick latency and hot-plate tests. Measurements were made $2 \mathrm{~h}$ (open bars) and $24 \mathrm{~h}$ (hatched bars) after treatment. Results are reported as average tail flick or hind paw lick or jump latency in seconds. Data are reported as the means \pm SEM. ${ }^{*} \mathrm{P}<0.05$ compared to anesthesia; ${ }^{* *} \mathrm{P}<$ 0.05 compared to removed soft tissue (RST); ${ }^{+} P<0.05$ compared to the anesthesia, RST and laminectomy groups (ANOVA followed by the Duncan test for all comparisons). For number of rats in each group, see Table 1. 
sponses to a stimulus of progressive pressure applied to the 3rd interdigital space of the left paw contralateral to the medullar hemisection.

A significant effect was observed on the right paw $\left(\mathrm{F}_{6,26}=14.49, \mathrm{P}<0.001\right)$, with a significant effect for the factor time $\left(\mathrm{F}_{6,26}=\right.$ $5.48, \mathrm{P}=0.027)$. Only marginal significance of time $\mathrm{x}$ lesion interaction $\left(\mathrm{F}_{6,26}=2.11, \mathrm{P}=\right.$ $0.086)$ was observed. The complete section and hemisection groups (the former at time 2 h) showed a significantly longer response
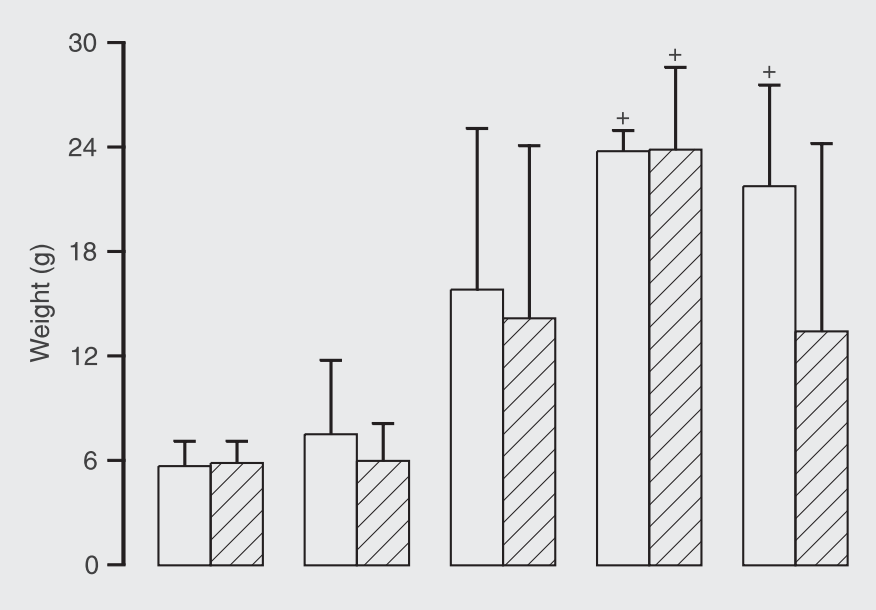

Right
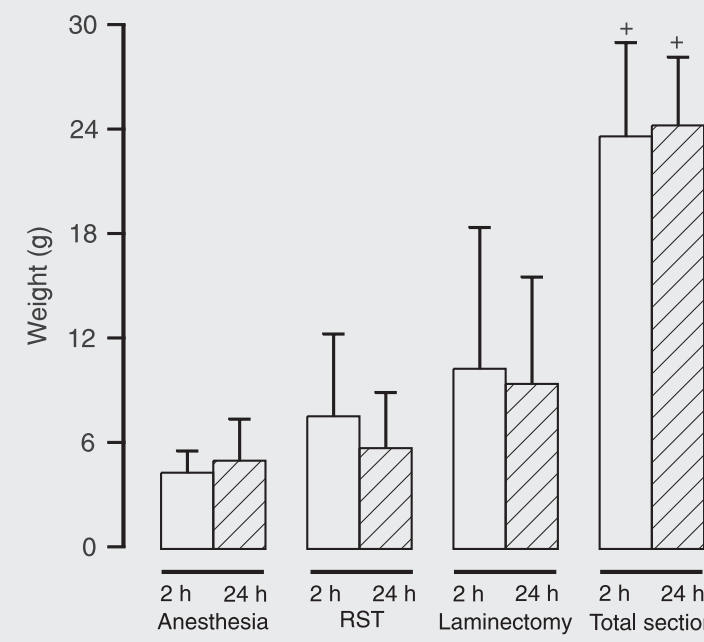

Left

Figure 3. Effects of spinal cord lesion on paw compression test on left and right paws. Measurements were made $2 \mathrm{~h}$ (open bars) and $24 \mathrm{~h}$ (hatched bars) after treatment. Results are reported as weight in $\mathrm{g}$. Data are reported as the means $\pm \mathrm{SEM} .{ }^{+} \mathrm{P}<0.05$ compared to other groups (ANOVA followed by the Duncan test). For number of rats in each group, see Table 1. time to the pain stimulus applied to the right paw ipsilateral to the medullar injury $(2 \mathrm{~h}$ : $\mathrm{F}_{6,26}=14.43, \mathrm{P}<0.001 ; 24 \mathrm{~h}: \mathrm{F}_{6,26}=9.97, \mathrm{P}$ $<0.001$ ).

A significant effect was observed on the left paw $\left(\mathrm{F}_{6,26}=3.45, \mathrm{P}=0.005\right)$; no significant effect of time $\left(\mathrm{F}_{6,26}=0.79, \mathrm{P}=0.38\right)$ or time $\mathrm{x}$ lesion interaction $\left(\mathrm{F}_{6,26}=0.64, \mathrm{P}=\right.$ $0.69)$ was observed. The complete section group showed a longer response time to the pain stimulus applied to the left paw ( 2 and $24 \mathrm{~h}: \mathrm{F}_{6,26}=1.05, \mathrm{P}=0.012$ ).

\section{Discussion}

The aim of the present study was to try to identify an SC injury process that could be useful for analyzing mechanisms of initial SC damage. The criteria we sought were reproducibility of changes in well-established sensory and motor tests 2 and $24 \mathrm{~h}$ after lesion. Our results show that motor scores were less sensitive than sensorimotor tests. A decreased ability to explore was detected in the animals 2 and $24 \mathrm{~h}$ after the procedures, but the hot-plate was the most sensitive test for detecting sensorimotor deficiencies following light, moderate or severe SC lesions. Laminectomy seemed to be a more drastic injury than vertebral compression. Hemisection induced medium to moderate injury and made it possible to quantify behavior and thereby to evaluate the consequences of the lesions. The results show that the locomotor scores and the open-field and hot-plate tests were reproducible, quantitative and sensitive tests for detecting functional deficiencies 2 and $24 \mathrm{~h}$ after SC lesion. The primary mechanisms implicated in SC damage may be investigated quantitatively at short times after injury ( 2 and $24 \mathrm{~h})$.

\section{Considerations on the behavioral tests}

The evaluation scales used in the present study detected significant deficiencies in the 
severe SC injury group. The Bohlman test score was 5 for all groups other than the hemisection and complete section groups and the test did not discriminate between lesion effects at 2 or $24 \mathrm{~h}$ despite the laminectomy procedure. The removed soft tissue and laminectomy groups presented score variations among animals of the same group and, in addition, between the median values corresponding to 2 and $24 \mathrm{~h}$. Hind limb motility scores were consistent for animals belonging to the anesthesia, complete section, hemisection, and vertebral compression groups, suggesting that the experimental procedures caused similar damage. This index indicated that laminectomy was more injurious to the animals than vertebral compression, suggesting sensitivity for the detection of light or moderate lesions.

The open-field test was sensitive to behavioral modifications and provided motor activity indexes for injured animals. This is a relatively simple, reproducible and quantitative test with enough sensitivity to detect functional deficiencies even in cases of light SC injury. Medium and severe SC injury caused a general reduction of exploratory activity after treatment. After $24 \mathrm{~h}$, the anesthesia and removed soft tissue groups presented an increase in the number of quadrants crossed. Interestingly, mild lesion induced by removing soft tissue, laminectomy, and vertebral compression, caused a greater decrease of rearing events $24 \mathrm{~h}$ after the procedure. Therefore, exploratory activity and rearing and grooming events were sensitive parameters for the detection of sensorimotor deficiencies and weaker responses 2 and $24 \mathrm{~h}$ after injury.

As a general effect, SC injury reduced spontaneous locomotor activity as measured in an open-field test. These results are in agreement with previous data reported by Basso et al. (21), Mills et al. (17), and Metz et al. (18). However, these investigators evaluated rats between 5 and 10 weeks after lesion. In addition, Metz et al. (18) reported that animals with low locomotion capacity presented a closer relationship between the 21-point open-field locomotion score (BBB scale) $(20,21)$ and activity in the open-field $(18,19,21)$. In addition, differences in exploratory behavior may reflect pain episodes (18). Mills et al. (17) showed that control animals on postsurgical 14, 28, and 60 days presented a reduction in exploratory activity, grooming and rearing events; in contrast, rats with SC injury demonstrated a decrease in the same parameters. These investigators suggested that spontaneous behaviors may be used to evaluate pain immediately after SC injury (18-21).

The hot-plate test was the most sensitive test of reflex function for the detection of sensorimotor deficiencies after injury. This test is also simple, reproducible and quantitative. The tail flick test showed similar sensitivity to the locomotion scales used in the present study (hind limb motility index, Bohlman score). The tail flick test reflects spinally organized reflexive responses, whereas the hot-plate test is thought to reflect supraspinal nociceptive processing. The paw compression test can be efficiently used in the hemisection model.

Thus, hind limb evaluation scores and spontaneous exploration events provided a sensitive index of the immediate effects of SC injury at 2 and $24 \mathrm{~h}$. The spontaneous locomotion, rearing and grooming events were the most sensitive parameters to detect sensorimotor deficiencies. The most sensitive test of reflex function was the hot-plate, which is also simple. In addition, the tail flick test had similar sensitivity to the Bohlman score and the paw compression test can be efficiently used in the hemisection lesion.

\section{Experimental spinal cord lesion}

Among the experimental models investigated in the present study, the hemisection model induced reproducible moderate SC injury which should allow us to quantitate some characteristics of the resulting behav- 
ior and analyze the evolution of the lesion and its consequences during the first $24 \mathrm{~h}$ after injury. Criticism of the hemisection and complete section models is based on the fact that, in addition to removal of the bone layer, the meninges are also cut $(4,12)$. In injury caused by crushing or contusion, although the bone layer is removed, the meninges are better preserved (10). Kwon et al. (22) suggested that sharp SC injury in which the cord is completely or partially transected is useful for assessing axonal regeneration anatomically. In contrast, blunt injury models in which the cord is compressed or contused more accurately mimic the typical human injury and provide a good setting for the study of secondary pathophysiologic processes immediately after injury. Our results showed that injury produced by vertebral compression was heterogeneous.

There is evidence that experimental SC injury in rats produces effects similar to those occurring in humans (13). In most patients traumatic injuries do not involve physical transection of the cord, but rather the SC is damaged by contusion, compression or stretch $(13,23)$. The lesion zone usually presented bleeding and damaged tissue $(1,2,11,12)$. Typically, residual white matter containing portions of the ascending sensory and motor tracts remains intact, allowing the possibility of recovery. However, during the first minutes and hours following injury, a secondary degenerative process is initiated by the primary mechanical injury that is proportional to the magnitude of the initial injury insult $(2,4,11,24)$. Nevertheless, the initial anatomical continuity of the injured SC in most cases, together with the present knowledge of many of the factors involved in the injury process, have led to the notion that treatments which interrupt the secondary cascade, if applied early, could improve SC tissue survival, and thus preserve the necessary anatomic substrate for functional recovery to take place. Promising research $(17,21,22,25,26)$ is being carried out to delineate the aspects that may be amenable to pharmacologic intervention. Few agents such as methylprednisolone (1, 12,23) have been subjected to large-scale human trials.

Considering the differences observed between sensitive and motor parameters and section and compression models, structural evaluation and/or biochemical assays could be helpful to explain these results. The extent of our understanding of immediate events after SC lesion may expand the neuroprotective strategies currently available.

The hemisection model was the one that permitted us to quantify the resulting behavior and to analyze the evolution of the lesion and its consequences during the first $24 \mathrm{~h}$.

\section{Acknowledgments}

The authors are indebted to S. Saltareli and E.C. Zieri for skillful technical assistance, and to Dr. M.A. Oliveira, and Professor F.S. Guimarães for the statistical analysis. We also thank Professor J.G. Nicholls (Neurobiology, Scuola Internazionale Superiore di Studi Avanzati, Trieste, Italy) for manuscript discussion and suggestions.

\section{References}

1. Dobkin BH, Havton LA. Basic advances and new avenues in therapy of spinal cord injury. Annu Rev Med 2004; 55: 255-282.

2. Hulsebosch CE. Recent advances in pathophysiology and treatment of spinal cord injury. Adv Physiol Educ 2002; 26: 238-255.

3. Nicholls J, Saunders N. Regeneration of immature mammalian spinal cord after injury. Trends Neurosci 1996; 19: 229-234.
4. Tator $\mathrm{CH}$, Fehlings MG. Review of the secondary injury theory of acute spinal cord trauma with emphasis on vascular mechanisms. $J$ Neurosurg 1991; 75: 15-26.

5. Schwab ME, Bartholdi D. Degeneration and regeneration of axons in the lesioned spinal cord. Physiol Rev 1996; 76: 319-370.

6. Noble LJ, Wrathall JR. Spinal cord contusion in the rat: morphomet- 
ric analyses of alterations in the spinal cord. Exp Neurol 1985; 88: 135-149.

7. Beattie MS, Bresnahan JC, Komon J, Tovar CA, Van Meter M, Anderson DK, et al. Endogenous repair after spinal cord contusion injuries in the rat. Exp Neurol 1997; 148: 453-463.

8. Rivlin AS, Tator $\mathrm{CH}$. Effect of duration of acute spinal cord compression in a new acute cord injury model in the rat. Surg Neurol 1978; 10: 38-43.

9. Gruner JA, Yee AK, Blight AR. Histological and functional evaluation of experimental spinal cord injury: evidence of a stepwise response to graded compression. Brain Res 1996; 729: 90-101.

10. Noble LJ, Ellison JA. Effect of transection on the blood-spinal cord barrier of the rat after isolation from descending sources. Brain Res 1989; 487: 299-310.

11. Dusart I, Schwab ME. Secondary cell death and the inflammatory reaction after dorsal hemisection of the rat spinal cord. Eur $J$ Neurosci 1994; 6: 712-724.

12. Basso DM. Behavioral testing after spinal cord injury: congruities, complexities, and controversies. J Neurotrauma 2004; 21: 395-404.

13. Rosenzweig ES, McDonald JW. Rodent models for treatment of spinal cord injury: research trends and progress toward useful repair. Curr Opin Neurol 2004; 17: 121-131.

14. Del-Bel EA, Borges CA, Defino HL, Guimaraes FS. Induction of Fos protein immunoreactivity by spinal cord contusion. Braz J Med Biol Res 2000; 33: 521-528.

15. Tarlov IM, Klinger $\mathrm{H}$, Vitale $\mathrm{S}$. Spinal cord compression studies. AMA Arch Neurol Psychiatry 1953; 71: 271-290.

16. Bohlman HH, Bahniuk E, Field G, Raskulinecz G. Spinal cord monitoring of experimental incomplete cervical spinal cord injury: a preliminary report. Spine 1981; 6: 428-436.

17. Mills CD, Grady JJ, Hulsebosch CE. Changes in exploratory behavior as a measure of chronic central pain following spinal cord injury.
J Neurotrauma 2001; 18: 1091-1105.

18. Metz GA, Merkler D, Dietz V, Schwab ME, Fouad K. Efficient testing of motor function in spinal cord injured rats. Brain Res 2000; 883: 165-177.

19. Randall LO, Selitto JJ. A method for measurement of analgesic activity on inflamed tissue. Arch Int Pharmacodyn Ther 1957; 111: 409-419.

20. Basso DM, Beattie MS, Bresnahan JC. Graded histological and locomotor outcomes after spinal cord contusion using the NYU weight-drop device versus transection. Exp Neurol 1996; 139: 244 256.

21. Basso DM, Beattie MS, Bresnahan JC, Anderson DK, Faden AI, Gruner JA, et al. MASCIS evaluation of open-field locomotor scores: effects of experience and teamwork on reliability. Multicenter Animal Spinal Cord Injury Study. J Neurotrauma 1996; 13: 343-359.

22. Kwon BK, Oxland TR, Tetzlaff W. Animal models used in spinal cord regeneration research. Spine 2002; 27: 1504-1510.

23. Bracken MB, Shepard MJ, Collins WF, Holford TR, Young W, Baskin DS, et al. A randomized, controlled trial of methylprednisolone or naloxone in the treatment of acute spinal-cord injury. Results of the Second National Acute Spinal Cord Injury Study. N Engl J Med 1990; 322: 1405-1411.

24. Kakulas BA. The clinical neuropathology of spinal cord injury. A guide to the future. Paraplegia 1987; 25: 212-216.

25. Wintzer M, Mladinic M, Lazarevic D, Casseler C, Cattaneo A, Nicholls J. Strategies for identifying genes that play a role in spinal cord regeneration. J Anat 2004; 204: 3-11.

26. Mladinic M, Wintzer M, Del Bel E, Casseler C, Lazarevic D, Crovella $S$, et al. Differential expression of genes at stages when regeneration can and cannot occur after injury to immature mammalian spinal cord. Cell Mol Neurobiol 2005; 25: 407-426. 